\title{
THE SCHUR MULTIPLIER OF CENTRAL PRODUCT OF GROUPS
}

\author{
SUMANA HATUI, L. R. VERMANI, AND MANOJ K. YADAV
}

\begin{abstract}
Let $G$ be a central product of two groups $H$ and $K$. We study second cohomology group of $G$, having coefficients in a divisible abelian group $D$ with trivial $G$-action, in terms of the second cohomology groups of certain quotients of $H$ and $K$. In particular, for $D=\mathbb{C}^{*}$, some of our results provide a refinement of results from [Some groups with non-trivial multiplicators, Math. Z. 120 (1971), 307-308] and [On the Schur multiplicator of a central quotient of a direct product of groups, J. Pure Appl. Algebra 3 (1973), 73-82].
\end{abstract}

\section{INTRODUCTION}

The Schur multiplier $\mathrm{M}(G)$ of a given group $G$, introduced by Schur in 1904 [6], is the second cohomology group $\mathrm{H}^{2}\left(G, \mathbb{C}^{*}\right)$ of $G$ with coefficients in $\mathbb{C}^{*}$. Let a finite group $G$ be the direct product of two groups $H$ and $K$. Then the formulation of the Schur multiplier of $G$ in terms of the Schur multipliers of $H$ and $K$ was given by Schur himself 7 . Such a formulation, when $G$ is a semidirect product of groups $H$ and $K$, was given by Tahara [8].

We say that $G$ is an internal central product of normal subgroups $H$ and $K$ amalgamating $A$ if $G=H K$ with $A=H \cap K$ and $[H, K]=1$. Let $H, K$ be two groups with isomorphic subgroups $A \leq \mathrm{Z}(H), B \leq \mathrm{Z}(K)$ under an isomorphism $\phi: A \rightarrow B$. Consider the normal subgroup $U=\left\{\left(a, \phi(a)^{-1}\right) \mid a \in A\right\}$. Then the group $G:=(H \times K) / U$ is called the external central product of $H$ and $K$ amalgamating $A$ and $B$ via $\phi$. The external central product $G$ can be viewed as an internal central product of the images of $H \times 1$ and $1 \times K$ in $G$. For this reason, we do not differentiate between external and internal central products, and consider only internal ones.

Let $G$ be a finite group which is a central product of subgroups $H$ and $K$ amalgamating $A$. Wiegold [10] proved that $\mathrm{M}(G)$ contains a subgroup isomorphic to $H / A \otimes K / A$, the abelian tensor product of $H / A$ and $K / A$. A generalization of this result for an arbitrary central quotient of direct product of two arbitrary groups was considered in [2].

Recall that $\mathrm{H}^{2}(G, A)$ denotes the second cohomology group of a group $G$ with coefficients in a $G$-module $A$. We are particularly interested in the case when $A$ is a trivial $G$-module and is divisible. We reserve $D$ for such a module. Throughout the paper, unless said otherwise explicitly, $G$ is always a central product of its normal subgroups $H$ and $K$ with $A=H \cap K$. In this paper we study $\mathrm{H}^{2}(G, D)$, in terms of the second cohomology groups of certain quotients of $H$ and $K$ with coefficients in $D$. Set $Z=H^{\prime} \cap K^{\prime}$, where $X^{\prime}$ denotes the commutator subgroup of a group $X$. The following result provides a reduction to the case when $Z=1$.

Theorem A. Let $B$ be a subgroup of $G$ such that $B \leq Z$. Then

$$
\mathrm{H}^{2}(G, D) \cong \mathrm{H}^{2}(G / B, D) / N,
$$

where $N \cong \operatorname{Hom}(B, D)$.

2010 Mathematics Subject Classification. 20J06.

Key words and phrases. Second cohomology group, Schur multiplier, central product.

The second author would like to thank Harish-Chandra Research Institute, Allahabad for the excellent hospitality provided to him couple of times during which a major part of this work was done. 
This result is very useful for computational purposes when $G$ is finite and $M(G / B)$ is known. Just to elaborate, we immediately get the following result for finite extraspecial $p$-groups proved in [1, Corollary 3.2].

Corollary 1.1. Let $G$ be an extra-special p-group of order $p^{2 n+1}, n \geq 2$. Then $M(G)$ is an elementary abelian p-group of order $p^{2 n^{2}-n-1}$.

By the tensor product $G_{1} \otimes G_{2}$ of two groups $G_{1}$ and $G_{2}$, we always mean the abelian tensor product, i.e., $G_{1} / G_{1}^{\prime} \otimes G_{2} / G_{2}^{\prime}$. Our next result is the following:

Theorem B. Let $L \cong \operatorname{Hom}\left(\left(A \cap H^{\prime}\right) / Z, D\right), M \cong \operatorname{Hom}\left(\left(A \cap K^{\prime}\right) / Z, D\right)$ and $N \cong$ $\operatorname{Hom}(Z, D)$. Then the following statements hold true:

(i) $\left(\mathrm{H}^{2}(H / A, D) / L \oplus \mathrm{H}^{2}(K / A, D) / M\right) / N \oplus \operatorname{Hom}(H / A \otimes K / A, D)$ embeds in $\mathrm{H}^{2}(G, D)$;

(ii) $\mathrm{H}^{2}(G, D)$ embeds in $\left(\mathrm{H}^{2}(H / Z, D) \oplus \mathrm{H}^{2}(K / Z, D)\right) / N \oplus \operatorname{Hom}(H \otimes K, D)$.

In particular, for $D=\mathbb{C}^{*}$, assertion (i) of Theorem B provides a refinement of results from [2] and [10].

On the way to proving these results, we obtain some commutative diagrams and exact sequences which might be of independent mathematical interest. Although our techniques are mostly cohomological, free presentation also shows up occasionally. In Section 2 we recall some known results and definitions, and establish a basic commutative diagram, which we refer to several times in what follows. Proofs of Theorems A and B are presented in Section 3. The final section contains several examples exhibiting various situations in which we determine whether or not any embedding in Theorem B actually becomes isomorphism.

\section{NotATIONS AND PRELIMINARIES}

Let $F / R$ be a free presentation of $X$ and $N$ be a normal subgroup of $X$. Let $S / R$ be the induced free presentation of $N$ for some subgroup $S$ of $F$. The following crucial result then follows from [9, Corollary 3.5].

Lemma 2.1. The inflation homomorphism inf : $\mathrm{H}^{2}(X / N, D) \rightarrow \mathrm{H}^{2}(X, D)$ is surjective if and only if $[F, R]=R \cap[F, S]$.

For an arbitrary group $X$ and a subgroup $N$, by $\operatorname{res}_{N}^{X}$ we denote the restriction homomorphism from $\operatorname{Hom}(X, D)$ to $\operatorname{Hom}(N, D)$ as well as the restriction homomorphism from $\mathrm{H}^{2}(X, D)$ to $\mathrm{H}^{2}(N, D)$. When the meaning is clear from the context, we write res for $\operatorname{res}_{N}^{X}$.

Let us consider the following central exact sequence for an arbitrary group $X$ and a central subgroup $N$ :

$$
1 \rightarrow N \rightarrow X \rightarrow X / N \rightarrow 1 .
$$

Then we get the exact sequence

$$
0 \rightarrow \operatorname{Hom}\left(N \cap X^{\prime}, D\right) \stackrel{\text { tra }}{\rightarrow} \mathrm{H}^{2}(X / N, D) \stackrel{\inf }{\rightarrow} \mathrm{H}^{2}(X, D) \stackrel{\chi}{\rightarrow} \mathrm{H}^{2}(N, D) \oplus \operatorname{Hom}(X \otimes N, D),
$$

where tra : $\operatorname{Hom}(N, D) \rightarrow \mathrm{H}^{2}(X / N, D)$ is the transgression homomorphism and $\chi=($ res, $\psi)$ as defined by Iwahori and Matsumoto [4]. To be more precise, res : $\mathrm{H}^{2}(X, D) \rightarrow \mathrm{H}^{2}(N, D)$ is the restriction homomorphism and $\psi: \mathrm{H}^{2}(X, D) \rightarrow \operatorname{Hom}(X \otimes N, D)$ is defined as $\psi(\xi)(\bar{x}, n)=f(x, n)-f(n, x)$ for all $\bar{x}=x X^{\prime} \in X / X^{\prime}$ and $n \in N$, where $\xi \in \mathrm{H}^{2}(X, D)$ and $f$ is a 2-cocycle representative of $\xi$.

Define a map

$$
\theta^{\prime}: \mathrm{H}^{2}(G, D) \rightarrow \mathrm{H}^{2}(H, D) \oplus \mathrm{H}^{2}(K, D) \oplus \operatorname{Hom}(H \otimes K, D)
$$

by $\theta^{\prime}=\left(\operatorname{res}_{H}^{G}, \operatorname{res}_{K}^{G}, \nu\right)$. Here $\nu: \mathrm{H}^{2}(G, D) \rightarrow \operatorname{Hom}(H \otimes K, D)$ is a homomorphism defined as follows. If $\xi \in \mathrm{H}^{2}(G, D)$ is represented by a 2-cocycle $f$, then $\nu(\xi)$ is the homomorphism $\bar{f} \in \operatorname{Hom}(H \otimes K, D)$ defined by $\bar{f}(\bar{h} \otimes \bar{k})=f(h, k)-f(k, h)$, where $\bar{h}=h H^{\prime}$ and $\bar{k}=k K^{\prime}$. It is now not difficult to see that $\theta^{\prime}$ is indeed a homomorphism. 
Consider the natural homomorphisms $\alpha: A H^{\prime} / H^{\prime} \otimes K \rightarrow H \otimes K, \beta: H \otimes A K^{\prime} / K^{\prime} \rightarrow$ $H \otimes K$, induced by obvious inclusion maps, and $\lambda: H \otimes K \rightarrow H / A \otimes K / A$, induced by natural projection. We now get the following exact sequence:

$$
\left(A H^{\prime} / H^{\prime} \otimes K\right) \oplus\left(H \otimes A K^{\prime} / K^{\prime}\right) \stackrel{\mu_{1}}{\rightarrow} H \otimes K \stackrel{\lambda}{\rightarrow} H / A \otimes K / A \rightarrow 0,
$$

where $\mu_{1}(x, y)=\alpha(x)+\beta(y)$.

We have natural epimorphisms $f: H \otimes A \rightarrow H \otimes A K^{\prime} / K^{\prime}$ and $g: A \otimes K \rightarrow A H^{\prime} / H^{\prime} \otimes K$. Consider the isomorphism $\eta: K \otimes A \rightarrow A \otimes K$, which, on the generators, is defined by $\eta(k \otimes a)=(a \otimes k)$. Using this, we have an epimorphism $(f, g \circ \eta):(H \otimes A) \oplus(K \otimes A) \rightarrow$ $\left(H \otimes A K^{\prime} / K^{\prime}\right) \oplus\left(A H^{\prime} / H^{\prime} \otimes K\right)$. Let $\mu=\mu_{1} \circ(f, g \circ \eta)$. Then $\operatorname{Im}\left(\mu_{1}\right)=\operatorname{Im}(\mu)$ and the above exact sequence leads to the exact sequence:

$$
(H \otimes A) \oplus(K \otimes A) \stackrel{\mu}{\rightarrow} H \otimes K \stackrel{\lambda}{\rightarrow} H / A \otimes K / A \rightarrow 0 .
$$

This exact sequence then gives the exact sequence

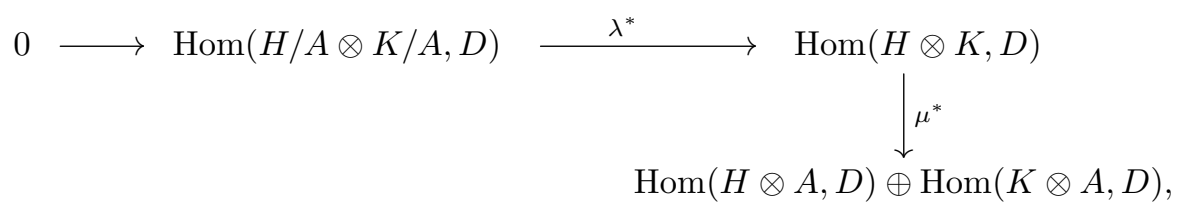

where the homomorphisms $\mu^{*}$ and $\lambda^{*}$ are induced by $\mu$ and $\lambda$ respectively.

Let $\alpha: H / H^{\prime} \oplus K / K^{\prime} \rightarrow G / G^{\prime}$ be the homomorphism induced by the inclusion maps $H \rightarrow G, K \rightarrow G$. Then $\alpha$ is clearly onto. Now $\alpha$ induces an epimorphism $\left(H / H^{\prime} \oplus\right.$ $\left.K / K^{\prime}\right) \otimes A \rightarrow G \otimes A$, which in turn induces a monomorphism $\alpha^{*}: \operatorname{Hom}(G \otimes A, D) \rightarrow$ $\operatorname{Hom}(H \otimes A, D) \oplus \operatorname{Hom}(K \otimes A, D)$. Let $\triangle: H^{2}(A, D) \rightarrow H^{2}(A, D) \oplus H^{2}(A, D)$ be defined by $\triangle(\xi)=(\xi, \xi)$ for $\xi \in H^{2}(A, D)$.

Set $\bar{G}=G / A, \bar{H}=H / A$ and $\bar{K}=K / A$. Let $\xi \in \mathrm{H}^{2}(\bar{G}, D)$ and $f$ be a 2-cocycle representing $\xi$. Recall that

$$
\theta: \mathrm{H}^{2}(\bar{G}, D) \rightarrow \mathrm{H}^{2}(\bar{H}, D) \oplus \mathrm{H}^{2}(\bar{K}, D) \oplus \operatorname{Hom}(\bar{H} \otimes \bar{K}, D)
$$

is the isomorphism defined by

$$
\theta(\xi)=\left(\operatorname{res}_{\bar{H}}^{\bar{G}}(\xi), \operatorname{res}_{\bar{K}}^{\bar{G}}(\xi), \nu_{1}(\xi)\right)
$$

where $\nu_{1}: \mathrm{H}^{2}(\bar{G}, D) \rightarrow \operatorname{Hom}(\bar{H} \otimes \bar{K}, D)$ is a homomorphism given by $\nu_{1}(\xi)(\tilde{h} \otimes \tilde{k})=f(h, k)-$ $f(k, h)$, with $h \in \bar{H}, k \in \bar{K}, \tilde{h}=h \bar{H}^{\prime}$ and $\tilde{k}=k \bar{K}^{\prime}$. Take $X_{1}=\mathrm{H}^{2}(A, D) \oplus \operatorname{Hom}(H \otimes A, D)$, $X_{2}=\mathrm{H}^{2}(A, D) \oplus \operatorname{Hom}(K \otimes A, D), X_{3}=\operatorname{Hom}(H \otimes A, D) \oplus \operatorname{Hom}(K \otimes A, D)$ and $Y=$ $\mathrm{H}^{2}(A, D) \oplus \mathrm{H}^{2}(A, D)$. We now get the following diagram (Diagram 1) with exact columns. In this diagram, for want of space, we suppress the use of $D$, i.e., we write $\operatorname{Hom}(X, D)$ as $\operatorname{Hom}(X)$ and $\mathrm{H}^{2}(X, D)$ as $\mathrm{H}^{2}(X)$ for a given group $X$. 


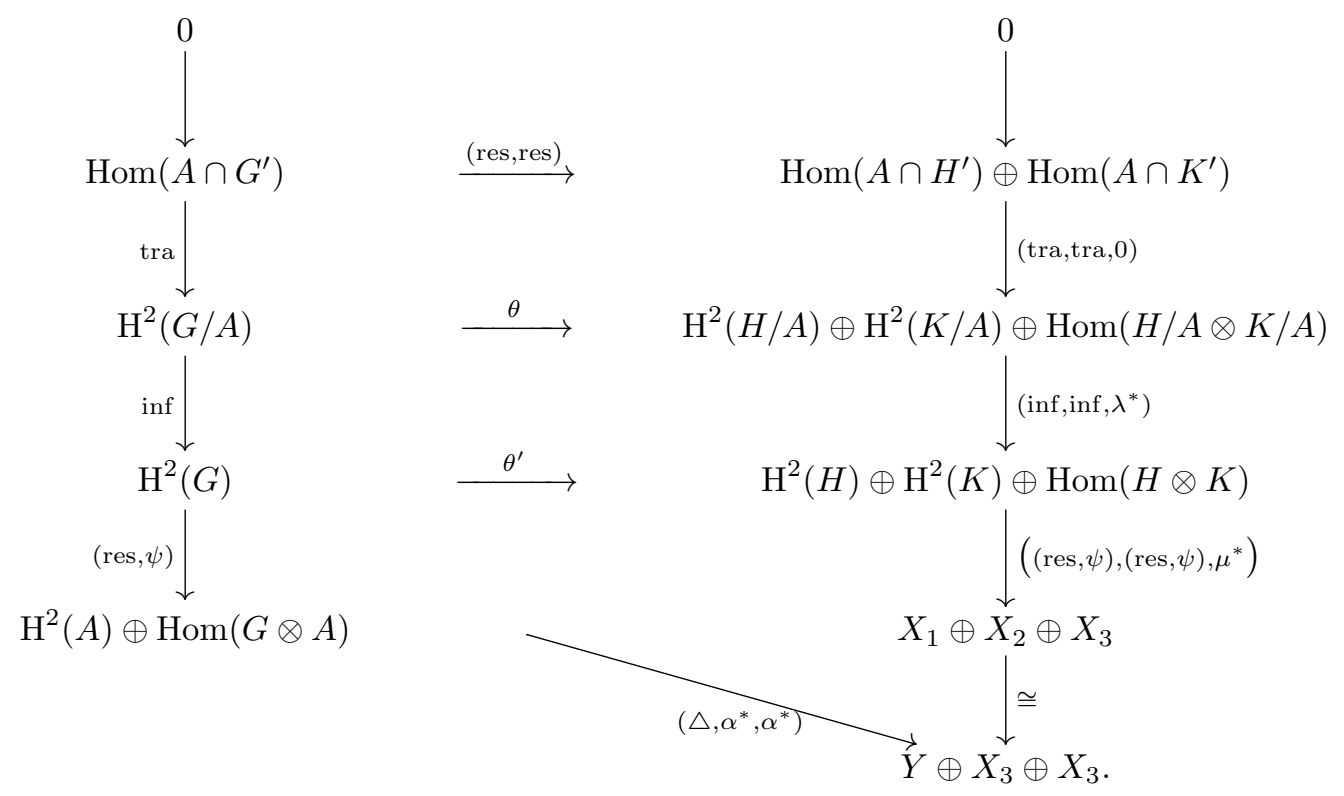

Diagram 1

Lemma 2.2. Diagram 1 is commutative.

Proof. It is a routine check to see that the topmost and middle rectangles are commutative. Observe that $\operatorname{res}_{A}^{H} \circ \operatorname{res}_{H}^{G}=\operatorname{res}_{A}^{G}$ and $\operatorname{res}_{A}^{K} \circ \operatorname{res}_{K}^{G}=\operatorname{res}_{A}^{G}$. It is also clear from the definitions that $\left(\alpha^{*}, \alpha^{*}\right) \circ \psi=\left((\psi, \psi), \mu^{*}\right) \circ \theta^{\prime}$. Thus it follows that the bottom part of the diagram is also commutative.

\section{Proofs}

In this section we present proofs of the results stated in the introduction. We start with the following result.

Theorem 3.1. For any central subgroup $B$ of $G$ contained in $H^{\prime} \cap K^{\prime}(=Z)$, the inflation homomorphism inf : $\mathrm{H}^{2}(G / B, D) \rightarrow \mathrm{H}^{2}(G, D)$ is surjective.

Proof. Let $F / R$ be a free presentation of $G$. Then the normal subgroups $H, K$ and $B$ can be freely presented as $S_{1} / R, S_{2} / R$ and $S / R$ respectively, where $S_{1}, S_{2}$ and $S$ are normal subgroups of $F$. Further, $Z \cong\left(S_{1}^{\prime} \cap S_{2}^{\prime}\right) R / R$. Note that $F=S_{1} S_{2}, S \subseteq\left(S_{1}^{\prime} \cap S_{2}^{\prime}\right) R$ and $\left[S_{1}, S_{2}\right] \subseteq R$.

By Lemma 2.1. it is enough to prove that $[F, R]=R \cap[F, S]$. Since $\left[S_{1}, S, S_{2}\right] \subseteq\left[S_{2}, S\right]$, we have

$$
\begin{aligned}
R \cap[F, S] & =[F, S]=\left[S_{1} S_{2}, S\right]=\left[S_{1}, S\right]\left[S_{1}, S, S_{2}\right]\left[S_{2}, S\right] \\
& =\left[S_{1}, S\right]\left[S_{2}, S\right] .
\end{aligned}
$$

Observe that $\left[S_{1}, S\right] \subseteq\left[S_{1}, S_{2}^{\prime} R\right]=\left[S_{1}, R\right]\left[S_{1}, S_{2}^{\prime}\right]\left[S_{1}, S_{2}^{\prime}, R\right]$. Since both $\left[S_{1}, S_{2}, S_{2}\right]$ and $\left[S_{2}, S_{1}, S_{2}\right]$ are contained in $[F, R]$, by the three subgroup lemma $\left[S_{1}, S_{2}^{\prime}\right] \subseteq[F, R]$. Hence $\left[S_{1}, S\right] \subseteq[F, R]$. Similarly $\left[S_{2}, S\right] \subseteq[F, R]$. Therefore $R \cap[F, S] \subseteq[F, R]$. Since $[F, R] \subseteq$ $R \cap[F, S]$, it follows that $[F, R]=R \cap[F, S]$, and the proof is complete. 
Proof of Theorem A. It follows from Theorem 3.1 that inf, in the following exact sequence, is surjective.

$$
0 \rightarrow \operatorname{Hom}(B, D) \stackrel{\text { tra }}{\rightarrow} \mathrm{H}^{2}(G / B, D) \stackrel{\text { inf }}{\rightarrow} \mathrm{H}^{2}(G, D) .
$$

Since $\operatorname{Hom}(B, D) \cong \operatorname{Im}(\operatorname{tra})=\operatorname{Ker}(\inf )$, the proof is complete.

We now mainly concentrate on the homomorphism $\theta^{\prime}$ defined in 2.1p. We start with the following result about the kernel of $\theta^{\prime}$.

Lemma 3.2. $\operatorname{Ker}\left(\theta^{\prime}\right)=\left\{\inf (\eta) \mid \eta \in \theta^{-1}(\operatorname{Im}(\right.$ tra, tra, 0$\left.))\right\}$.

Proof. Let $\xi \in \operatorname{ker}\left(\theta^{\prime}\right)$. By the commutativity of the bottommost part of Diagram 1, it follows that $\left(\triangle, \alpha^{*}, \alpha^{*}\right)\left(\operatorname{res}_{A}^{G}, \psi\right)(\xi)=0$. Since $\alpha^{*}$ is a monomorphism and $\triangle=(\mathrm{Id}, \mathrm{Id})$, it follows that $\left(\operatorname{res}_{A}^{G}, \psi\right)(\xi)=0$. Now the existence of $\eta \in \mathrm{H}^{2}(G / A, D)$ such that $\xi=\inf (\eta)$ is guaranteed by the exactness of the left column in Diagram 1. Thus $\operatorname{Ker}\left(\theta^{\prime}\right) \subseteq \operatorname{Im}(\inf$ : $\left.\mathrm{H}^{2}(G / A, D) \rightarrow \mathrm{H}^{2}(G, D)\right)$.

By the commutativity of the middle rectangle of Diagram 1, it follows that

$$
0=\theta^{\prime}(\xi)=\theta^{\prime}(\inf (\eta))=\theta^{\prime} \circ \inf (\eta)=\left(\inf , \inf , \lambda^{*}\right) \circ \theta(\eta) .
$$

Again invoking Diagram 1, we get $\theta(\eta) \in \operatorname{Im}($ tra, tra, 0$)$. Hence $\eta \in \theta^{-1}(\operatorname{Im}(\operatorname{tra}, \operatorname{tra}, 0))$. That $\theta^{\prime}(\inf (\eta))=0$ for $\eta \in \theta^{-1}(\operatorname{Im}(\operatorname{tra}$, tra, 0$))$ follows from the commutativity of Diagram 1 with the right column exact. This completes the proof.

We have an exact sequence

$$
0 \rightarrow H^{\prime} \cap K^{\prime} \stackrel{\alpha_{1}}{\longrightarrow}\left(A \cap H^{\prime}\right) \oplus\left(A \cap K^{\prime}\right) \stackrel{\alpha_{2}}{\longrightarrow} A \cap G^{\prime} \rightarrow 0,
$$

which induces an exact sequence

$$
0 \rightarrow \operatorname{Hom}\left(A \cap G^{\prime}, D\right) \stackrel{\alpha_{2}^{*}}{\longrightarrow} \operatorname{Hom}\left(A \cap H^{\prime}, D\right) \oplus \operatorname{Hom}\left(A \cap K^{\prime}, D\right) \stackrel{\alpha_{1}^{*}}{\longrightarrow} \operatorname{Hom}(Z, D) \rightarrow 0,
$$

in which $\alpha_{2}^{*}$ is the homomorphism (res, res).

The homomorphism $\alpha_{1}^{*}$ being surjective, for any $f \in \operatorname{Hom}(Z, D)$, there exists $g \in \operatorname{Hom}(A \cap$ $\left.H^{\prime}, D\right) \oplus \operatorname{Hom}\left(A \cap K^{\prime}, D\right)$ such that $f=\alpha_{1}^{*}(g)$. Let $g_{1} \in \operatorname{Hom}\left(A \cap H^{\prime}, D\right) \oplus \operatorname{Hom}\left(A \cap K^{\prime}, D\right)$ be another element such that $f=\alpha_{1}^{*}\left(g_{1}\right)$. Then there exists $\nu \in \operatorname{Hom}\left(A \cap G^{\prime}, D\right)$ such that $g-g_{1}=\alpha_{2}^{*}(\nu)$. For the convenience of writing, set $\zeta=\inf \circ \theta^{-1}$ (recall that $\theta$ is an isomorphism). Now, using the commutativity of the topmost rectangle of Diagram 1, we get

$$
\begin{aligned}
\zeta \circ(\operatorname{tra}, \operatorname{tra}, 0)(g) & =\zeta \circ(\operatorname{tra}, \operatorname{tra}, 0)\left(g_{1}\right)+\zeta \circ(\operatorname{tra}, \operatorname{tra}, 0)\left(\alpha_{2}^{*}(\nu)\right) \\
& =\zeta \circ(\operatorname{tra}, \operatorname{tra}, 0)\left(g_{1}\right)+\zeta \circ \theta \circ \operatorname{tra}(\nu) \\
& =\zeta \circ(\operatorname{tra}, \operatorname{tra}, 0)\left(g_{1}\right) .
\end{aligned}
$$

Hence $\zeta \circ($ tra, tra, 0$)$ is independent of the choice of $g \in \operatorname{Hom}\left(A \cap H^{\prime}, D\right) \oplus \operatorname{Hom}\left(A \cap K^{\prime}, D\right)$ with $\alpha_{1}^{*}(g)=f$. Setting $\chi(f)=\zeta \circ(\operatorname{tra}$, tra, 0$)$, we get a well defined map $\chi: \operatorname{Hom}(Z, D) \rightarrow$ $\mathrm{H}^{2}(G, D)$. It is now clear that $\chi$ is a homomorphism.

Theorem 3.3. The following sequence is exact:

$$
0 \rightarrow \operatorname{Hom}(Z, D) \stackrel{\chi}{\rightarrow} \mathrm{H}^{2}(G, D) \stackrel{\theta^{\prime}}{\rightarrow} \mathrm{H}^{2}(H, D) \oplus \mathrm{H}^{2}(K, D) \oplus \operatorname{Hom}(H \otimes K, D) .
$$

Proof. Suppose that $f \in \operatorname{Hom}(Z, D)$ and $\chi(f)=0$. Then $\inf \circ \theta^{-1} \circ(\operatorname{tra}, \operatorname{tra}, 0)(g)=0$ for some $g \in \operatorname{Hom}\left(A \cap H^{\prime}, D\right) \oplus \operatorname{Hom}\left(A \cap K^{\prime}, D\right)$ such that $f=\alpha_{1}^{*}(g)$. Thus there exists $\eta \in \operatorname{Hom}\left(A \cap G^{\prime}, D\right)$ such that $\theta^{-1} \circ(\operatorname{tra}, \operatorname{tra}, 0)(g)=\operatorname{tra}(\eta)$ by the commutativity of Diagram 1. Then $(\operatorname{tra}, \operatorname{tra}, 0)(g)=\theta \circ \operatorname{tra}(\eta)=(\operatorname{tra}, \operatorname{tra}, 0) \circ($ res, res $)(\eta)=(\operatorname{tra}, \operatorname{tra}, 0) \circ \alpha_{2}^{*}(\eta)$. Since (tra, tra, 0 ) is a monomorphism, we have $g=\alpha_{2}^{*}(\eta)$. Thus $f=\alpha_{1}^{*} \circ \alpha_{2}^{*}(\eta)=0$, which, $f$ being an arbitrary element, proves that $\chi$ is a monomorphism. That $\operatorname{Im}(\chi)=\operatorname{Ker}\left(\theta^{\prime}\right)$ is now clear from Lemma 3.2, and the proof is complete. 
The following is an immediate consequence of the preceding theorem.

Corollary 3.4. If $Z=1$, then

$$
\theta^{\prime}: \mathrm{H}^{2}(G, D) \rightarrow \mathrm{H}^{2}(H, D) \oplus \mathrm{H}^{2}(K, D) \oplus \operatorname{Hom}(H \otimes K, D)
$$

is a monomorphism.

Using the argument as in Theorem 3.3 and the observation that

$$
\inf \left(\theta^{-1}(\operatorname{Hom}(H / A \otimes K / A, D))\right) \cap \operatorname{ker}\left(\theta^{\prime}\right)=\{0\},
$$

which follows from the commutativity of the middle rectangle of Diagram 1, we get:

Corollary 3.5. The following sequence is exact

$0 \rightarrow \operatorname{Hom}(Z, D) \oplus \operatorname{Hom}(H / A \otimes K / A, D) \stackrel{\left(\chi, \inf \circ \theta^{-1}\right)}{\longrightarrow} \mathrm{H}^{2}(G, D) \stackrel{\text { (res,res) }}{\longrightarrow} \mathrm{H}^{2}(H, D) \oplus \mathrm{H}^{2}(K, D)$.

In particular, $\operatorname{Hom}(Z, D) \oplus \operatorname{Hom}(H / A \otimes K / A, D)$ embeds in $\mathrm{H}^{2}(G, D)$.

As we know by Theorem A that $\operatorname{Hom}(Z, D)$ embeds in $\mathrm{H}^{2}(G / Z, D)$. We now prove a much stronger result in the following

Theorem 3.6. $\operatorname{Hom}(Z, D)$ embeds in $\mathrm{H}^{2}(H / A, D) / L \oplus \mathrm{H}^{2}(K / A, D) / M$, where $L \cong \operatorname{Hom}((A \cap$ $\left.\left.H^{\prime}\right) / Z, D\right)$ and $M \cong \operatorname{Hom}\left(\left(A \cap K^{\prime}\right) / Z, D\right)$.

Proof. Let $\alpha: \operatorname{Hom}\left(A \cap G^{\prime}, D\right) \rightarrow \operatorname{Hom}(Z, D)$ be the epimorphism induced by the inclusion $Z \hookrightarrow A \cap G^{\prime}$. Set $Y_{1}=\operatorname{Im}\left(\inf : \mathrm{H}^{2}(G / A, D) \rightarrow \mathrm{H}^{2}(G / Z, D)\right)$. Since $G / Z$ is a central product of $H / Z$ and $K / Z$ with $(H / Z)^{\prime} \cap(K / Z)^{\prime}=1$, it follows that $Y_{1}$ is isomorphic to $\mathrm{H}^{2}(H / A, D) / L \oplus \mathrm{H}^{2}(K / A, D) / M \oplus \operatorname{Hom}(H / A \otimes K / A, D)$, where $L \cong \operatorname{Hom}\left(\left(A \cap H^{\prime}\right) / Z, D\right)$ and $M \cong \operatorname{Hom}\left(\left(A \cap K^{\prime}\right) / Z, D\right)$.

Consider the following commutative diagram (with rows not necessarily exact):

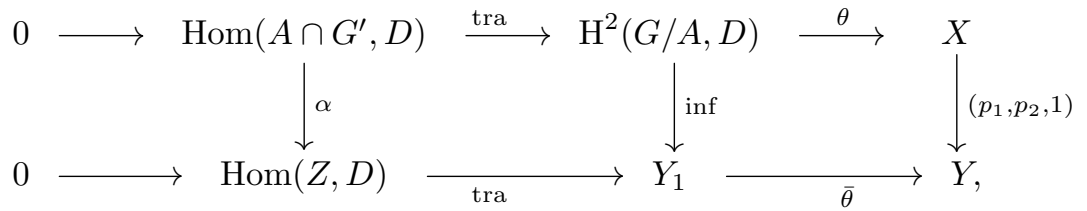

where

$$
\begin{gathered}
X=\mathrm{H}^{2}(H / A, D) \oplus \mathrm{H}^{2}(K / A, D) \oplus \operatorname{Hom}(H / A \otimes K / A, D), \\
Y=\mathrm{H}^{2}(H / A, D) / L \oplus \mathrm{H}^{2}(K / A, D) / M \oplus \operatorname{Hom}(H / A \otimes K / A, D),
\end{gathered}
$$

$\bar{\theta}$ is an isomorphism and $p_{i}, i=1,2$, are natural projections.

Let $\beta \in \operatorname{Hom}(Z, D)$. Then there exists $\bar{\beta} \in \operatorname{Hom}\left(A \cap G^{\prime}, D\right)$ such that $\beta=\alpha(\bar{\beta})$. Let $\operatorname{tra}(\bar{\beta})=\xi \in \mathrm{H}^{2}(G / A, D)$. The element $\xi$ is represented by a 2-cocycle $f$ given by

$$
f(\bar{x}, \bar{y})=\bar{\beta}\left(\mu(\bar{x}) \mu(\bar{y}) \mu(\bar{x} \bar{y})^{-1}\right), \quad \bar{x}=x A \in G / A \text { and } \bar{y}=y A \in G / A,
$$

where $\mu$ represents the section $\mu: G / A \rightarrow G$ in the exact sequence $1 \rightarrow A \rightarrow G \rightarrow G / A \rightarrow 1$.

Recall that $\theta=$ (res, res, $\nu_{1}$ ), where $\nu_{1}(\xi): H / A \otimes K / A \rightarrow D$ for $\bar{h}=h A \in H / A$ and $\bar{k}=k A \in K / A$ is given by

$$
\nu_{1}(\xi)\left(\bar{h}(H / A)^{\prime}, \bar{k}(K / A)^{\prime}\right)=f(\bar{h}, \bar{k})-f(\bar{k}, \bar{h}) .
$$

Plugging in the value of $f$ we have

$$
\begin{aligned}
\nu_{1}(\xi)\left(\bar{h}(H / A)^{\prime}, \bar{k}(K / A)^{\prime}\right) & =f(\bar{h}, \bar{k})-f(\bar{k}, \bar{h}) \\
& =\bar{\beta}\left(\mu(\bar{h}) \mu(\bar{k}) \mu(\bar{h} \bar{k})^{-1}\right)-\bar{\beta}\left(\mu(\bar{k}) \mu(\bar{h}) \mu(\bar{k} \bar{h})^{-1}\right) \\
& =\bar{\beta}\left(\mu(\bar{h}) \mu(\bar{k}) \mu(\bar{h} \bar{k})^{-1} \mu(\bar{k} \bar{h}) \mu(\bar{h})^{-1} \mu(\bar{k})^{-1}\right) \\
& =\bar{\beta}\left(\mu(\bar{h}) \mu(\bar{k}) \mu(\bar{h} \bar{k})^{-1} \mu(\bar{h} \bar{k}) \mu(\bar{k})^{-1} \mu(\bar{h})^{-1}\right) \\
& =0 .
\end{aligned}
$$


Hence $\theta(\operatorname{tra}(\bar{\beta})) \in \mathrm{H}^{2}(H / A, D) \oplus \mathrm{H}^{2}(K / A, D)$. That $\bar{\theta}(\operatorname{tra}(\beta)) \in \mathrm{H}^{2}(H / A, D) / L \oplus \mathrm{H}^{2}(K / A, D) / M$ now follows by the commutativity of the above diagram, which completes the proof.

Using an argument similar to one as in the preceding proof, we can also prove

Theorem 3.7. $\operatorname{Hom}(Z, D)$ embeds in $\mathrm{H}^{2}(H / Z, D) \oplus \mathrm{H}^{2}(K / Z, D)$.

The following is now an immediate consequence of Theorem A and the preceding theorem.

Corollary 3.8. If $A=Z$, then

$$
\mathrm{H}^{2}(G, D) \cong\left(\mathrm{H}^{2}(H / Z, D) \oplus \mathrm{H}^{2}(K / Z, D)\right) / \operatorname{Hom}(Z, D) \oplus \operatorname{Hom}(H / Z \otimes K / Z, D) .
$$

We are now ready to prove Theorem B.

Proof of Theorem B. As already observed in the proof of Theorem 3.6. $\operatorname{Im}\left(\mathrm{inf}: \mathrm{H}^{2}(G / A, D) \rightarrow\right.$ $\left.\mathrm{H}^{2}(G / Z, D)\right)$ is isomorphic to $\mathrm{H}^{2}(H / A, D) / L \oplus \mathrm{H}^{2}(K / A, D) / M \oplus \operatorname{Hom}(H / A \otimes K / A, D)$. The first assertion now follows from Theorem $\mathrm{A}$ and Theorem 3.6 .

The second assertion follows from Theorem A, Corollary 3.4 (with $G$ replaced by $G / Z$ ) and Theorem 3.7 .

It is perhaps an appropriate place to remark that

$$
\mathrm{H}^{2}(G, D) \cong\left(\mathrm{H}^{2}(H / A, D) / L \oplus \mathrm{H}^{2}(K / A, D) / M\right) / N \oplus \operatorname{Hom}(H / A \otimes K / A, D)
$$

if and only if inf : $\mathrm{H}^{2}(G / A, D) \rightarrow \mathrm{H}^{2}(G / Z, D)$ is an epimorphism, where $L, M$ and $N$ are as defined above.

The following result is immediate from the commutativity of the bottommost part of Diagram 1.

Proposition 3.9. Let $\xi \in \mathrm{H}^{2}(\bar{G}, D)$ such that $\theta^{\prime}(\xi)=\left(\xi_{1}, \xi_{2}, t\right)$, where $\bar{G}=G / Z$. Further, let either $\operatorname{res}_{\bar{A}} \bar{H}\left(\xi_{1}\right)=0$ or $\operatorname{res}_{\bar{A}} \bar{K}\left(\xi_{2}\right)=0$. Then the following statements are equivalent:

(i) $\xi \in \operatorname{Im}\left(\inf : \mathrm{H}^{2}(\bar{G} / \bar{A}, D) \rightarrow \mathrm{H}^{2}(\bar{G}, D)\right)$;

(ii) $\mu^{*}(t)=0$;

(iii) $\psi\left(\xi_{1}\right)=\psi\left(\xi_{2}\right)=0$.

As a consequence we have

Corollary 3.10. If inf $: \mathrm{H}^{2}(H / A, D) \rightarrow \mathrm{H}^{2}(H / Z, D)$ and inf $: \mathrm{H}^{2}(K / A, D) \rightarrow \mathrm{H}^{2}(K / Z, D)$ are epimorphisms, then

$$
H^{2}(G, D) \cong\left(H^{2}(H / Z, D) \oplus H^{2}(K / Z, D)\right) / \operatorname{Hom}(Z, D) \oplus \operatorname{Hom}(H / A \otimes K / A, D) .
$$

More precisely, the first embedding in Theorem B is an isomorphism.

Using Proposition 3.9, we also have

Theorem 3.11. If the second embedding in Theorem B is an isomorphism, then so is the first.

Proof. Since the isomorphism

$$
\mathrm{H}^{2}(G, D) \cong\left(\mathrm{H}^{2}(H / Z, D) \oplus \mathrm{H}^{2}(K / Z, D)\right) / \operatorname{Hom}(Z, D) \oplus \operatorname{Hom}(H / Z \otimes K / Z, D)
$$


is induced by the monomorphism $\theta^{\prime}$ as defined in 2.1 with $G$ replaced by $G / Z$, it follows from the commutative diagram

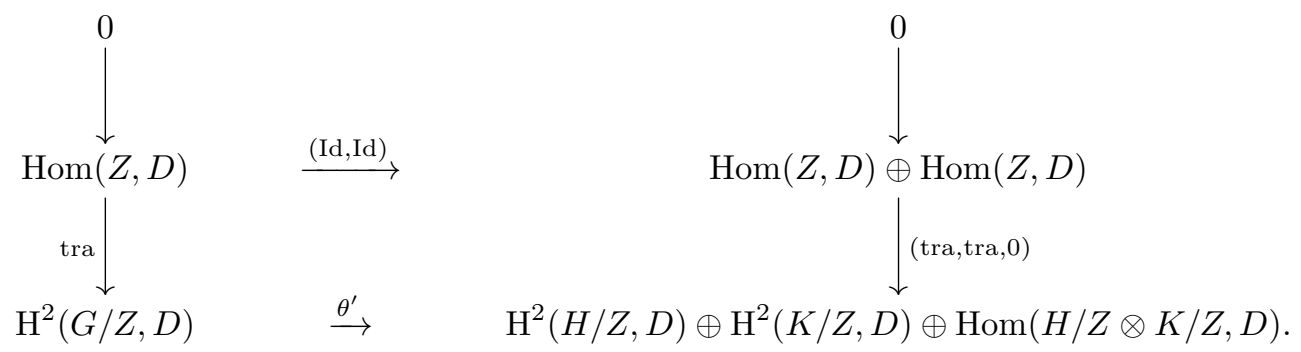

that $\theta^{\prime}$ is an isomorphism. Let $t \in \operatorname{Hom}(H / Z \otimes K / Z, D)$. Then there exists $\xi \in \mathrm{H}^{2}(G / Z, D)$ such that $\theta^{\prime}(\xi)=(0,0, t)$. It then follows from Diagram 1 (for $G / Z$ in place of $G$ ) that $($ res, $\psi)(\xi)=0$. By Proposition 3.9 we then have $\mu^{*}(t)=0$, which shows that $\lambda^{*}$ : $\operatorname{Hom}(H / A \otimes K / A, D) \rightarrow \operatorname{Hom}(H / Z \otimes K / Z, D)$ is an epimorphism, and hence, an isomorphism. Similarly, considering the elements $\xi_{1} \in \mathrm{H}^{2}(H / Z, D)$ and $\xi_{2} \in \mathrm{H}^{2}(K / Z, D)$ in succession, the above argument also shows that inf: $\mathrm{H}^{2}(H / A, D) \rightarrow \mathrm{H}^{2}(H / Z, D)$ and inf : $\mathrm{H}^{2}(K / A, D) \mathrm{H}^{2}(K / Z, D)$ are epimorphisms. Hence $\mathrm{H}^{2}(H / A, D) / L \cong \mathrm{H}^{2}(H / Z, D)$ and $\mathrm{H}^{2}(K / A, D) / M \cong \mathrm{H}^{2}(K / Z, D)$. It now follows that the first embedding in Theorem $\mathrm{B}$ is an isomorphism.

We conclude this section with the following remark made by J. Wiegold while reviewing 2 for AMS (see MR0349854 (50\#2347)). Let $G$ be the direct product $G=H \times K$ of its normal subgroups $H$ and $K$, and $U$ be an arbitrary central subgroup of $G$. Then $G / U$ can be viewed as a central product of $H U / U$ and $K U / U$. Thus all the above results make sense for $\mathrm{H}^{2}(G / U, D)$.

\section{EXAMPLES}

In this section we present several examples (all for finite $p$-groups) to show that various situations of Theorem B can indeed occur. By $\mathbb{Z}_{p}^{(n)}$, we denote the elementary abelian $p$ group of rank $n$, where $n \geq 1$. We start with an example which shows that neither of the two embeddings of Theorem B is necessarily an isomorphism.

Example 1. Let $H$ be the extraspecial $p$-groups of order $p^{3}$ and exponent $p$ and $K=\mathbb{Z}_{p}^{(n+1)}$, where $n \geq 1$. Let $G$ be a central product of $H$ and $K$ amalgamated at $A \cong H^{\prime} \cong \mathbb{Z}_{p}$. Note that $G=H \times \mathbb{Z}_{p}^{(n)}$. It is easy to see that

$$
\mathrm{M}(G) \cong \mathbb{Z}_{p}^{\left(\frac{1}{2} n(n+3)+2\right)} .
$$

Note that $Z=H^{\prime} \cap K^{\prime}=1$. Then

$$
\mathrm{M}(H / A) / \operatorname{Hom}\left(A \cap H^{\prime}, \mathbb{C}^{*}\right) \oplus \mathrm{M}(K / A) / \operatorname{Hom}\left(A \cap K^{\prime}, \mathbb{C}^{*}\right) \oplus \operatorname{Hom}\left(H / A \otimes K / A, \mathbb{C}^{*}\right)
$$

is isomorphic to $\mathbb{Z}_{p}^{\left(\frac{1}{2} n(n+3)\right)}$, which is strictly contained in $M(G)$. Since

$$
\mathrm{M}(H) \oplus \mathrm{M}(K) \oplus \operatorname{Hom}\left(H \otimes K, \mathbb{C}^{*}\right) \cong \mathbb{Z}_{p}^{\left(\frac{1}{2}(n+1)(n+4)+2\right)},
$$

it properly contains $M(G)$.

Before proceeding further, we mention the following interesting result by M. R. Jones 5 , Theorem 4.1(i)]. 
Theorem 4.1. Let $X$ be a finite group and $N$ a central subgroup. Then

$$
|\mathrm{M}(X)|\left|X^{\prime} \cap N\right| \text { divides }|\mathrm{M}(X / N)||\mathrm{M}(N)||(X / N) \otimes N| \text {. }
$$

The following two examples show that the first embedding in Theorem B can very well be an isomorphisms, but the second one can still be strict (i.e., not an isomorphism).

Example 2. Consider the group $G$ presented as

$$
G=\left\langle\alpha, \alpha_{1}, \alpha_{2}, \gamma \mid\left[\alpha_{1}, \alpha\right]=\gamma^{p^{2}}=\alpha_{2}, \alpha^{p}=\alpha_{1}^{p}=\alpha_{2}^{p}=1\right\rangle .
$$

Take $H=\left\langle\alpha, \alpha_{1}, \alpha_{2} \mid\left[\alpha_{1}, \alpha\right]=\alpha_{2}, \alpha^{p}=\alpha_{1}^{p}=\alpha_{2}^{p}=1\right\rangle$ and $K=\langle\gamma\rangle \cong \mathbb{Z}_{p^{3}}$. Then $G$ is a central product of $H$ and $K$ amalgamated at $A \cong\left\langle\alpha_{2}\right\rangle \cong\left\langle\gamma^{p^{2}}\right\rangle$. Note that $Z=1$ and

$$
\mathrm{M}(H / A) / \operatorname{Hom}\left(A \cap H^{\prime}, \mathbb{C}^{*}\right) \oplus \mathrm{M}(K / A) / \operatorname{Hom}\left(A \cap K^{\prime}, \mathbb{C}^{*}\right) \oplus \operatorname{Hom}\left(H / A \otimes K / A, \mathbb{C}^{*}\right)
$$

is isomorphic to $\mathbb{Z}_{p}^{(2)}$. By Theorem 4.1. we have $|\mathrm{M}(G)| \leq p^{2}$. Hence $\mathrm{M}(G) \cong \mathbb{Z}_{p}^{(2)}$, and therefore the first embedding in Theorem $\mathrm{B}$ is an isomorphisms. It is easy to see that

$$
\mathrm{M}(H) \oplus \mathrm{M}(K) \oplus \operatorname{Hom}\left(H \otimes K, \mathbb{C}^{*}\right) \cong \mathbb{Z}_{p}^{(4)},
$$

which shows that the second embedding is strict.

Example 3. Consider the group $G$ presented as

$$
G=\left\langle\alpha, \alpha_{1}, \alpha_{2}, \alpha_{3}, \gamma \mid\left[\alpha_{1}, \alpha\right]=\alpha_{2},\left[\alpha_{2}, \alpha\right]=\gamma^{p}=\alpha_{3}, \alpha^{p}=\alpha_{i}^{(p)}=1, i=1,2,3\right\rangle .
$$

Take $H=\left\langle\alpha, \alpha_{1}, \alpha_{2}, \alpha_{3} \mid\left[\alpha_{1}, \alpha\right]=\alpha_{2},\left[\alpha_{2}, \alpha\right]=\alpha_{3}, \alpha^{p}=\alpha_{i}^{(p)}=1, i=1,2,3\right\rangle$ and $K=$ $\langle\gamma\rangle \cong \mathbb{Z}_{p^{2}}$. Then $G$ is a central product of $H$ and $K$ amalgamated at $A \cong\left\langle\alpha_{3}\right\rangle \cong\left\langle\gamma^{p}\right\rangle$ and $Z=1$. Note that

$$
M(H / A) / \operatorname{Hom}\left(A \cap H^{\prime}, \mathbb{C}^{*}\right) \oplus M(K / A) / \operatorname{Hom}\left(A \cap K^{\prime}, \mathbb{C}^{*}\right) \oplus \operatorname{Hom}\left(H / A \otimes K / A, \mathbb{C}^{*}\right)
$$

is isomorphic to $\mathbb{Z}_{p}^{(3)}$, which embeds in $\mathrm{M}(G)$. Again by Theorem 4.1. we have $|\mathrm{M}(G)| \leq p^{3}$. Hence the first embedding is an isomorphism. That the second one is not can be easily seen as in Example 2.

We finally present an example which shows that both the embeddings in Theorem B can be isomorphisms.

Example 4. Let $H$ be the extraspecial $p$-groups of order $p^{3}$ and exponent $p^{2}$ and $K \cong \mathbb{Z}_{p^{n+1}}$, the cyclic group of order $p^{n+1}$, where $n \geq 1$. Let $G$ be a central product of $H$ and $K$ amalgamated at $A \cong H^{\prime} \cong \mathbb{Z}_{p}$. Note that $G=H \times \mathbb{Z}_{p^{n}}$. It is easy to see that $\mathrm{M}(G) \cong \mathbb{Z}_{p}^{(2)}$. Note that $Z=H^{\prime} \cap K^{\prime}=1$. Then

$$
\mathrm{M}(H / A) / \operatorname{Hom}\left(A \cap H^{\prime}, \mathbb{C}^{*}\right) \oplus \mathrm{M}(K / A) / \operatorname{Hom}\left(A \cap K^{\prime}, \mathbb{C}^{*}\right) \oplus \operatorname{Hom}\left(H / A \otimes K / A, \mathbb{C}^{*}\right)
$$

is isomorpic to $\mathbb{Z}_{p}^{(2)}$. Also

$$
\mathrm{M}(H) \oplus \mathrm{M}(K) \oplus \operatorname{Hom}\left(H \otimes K, \mathbb{C}^{*}\right) \cong \mathbb{Z}_{p}^{(2)} .
$$

Hence both the embeddings are isomorphisms.

\section{REFERENCES}

[1] N. Blackburn, and L. Evens, Schur multipliers of p-groups, J. Reine Angew. Math, 309 (1979), 100113.

[2] B. Eckmann, P. J. Hilton, and U. Stammbach, On the Schur multiplicator of a central quotient of a direct product of groups, J. Pure Appl. Algebra 3 (1973), 73-82.

[3] T. Ganea, , Homologie et extensions centrales de groupes, C.R. Acad, Sci., Paris, 266 (1968), 556-568.

[4] N. Iwahori, and H. Matsumoto, Several remarks on projective representations of finite groups, J. Fac. Sci. Univ. Tokyo, Sect. I, 10 (1964), 129-146. 
[5] M. R. Jones, Some inequalities for multiplicators of a finite group, Proc. Amer. Math. Soc. 39(3) (1973), 450-456.

[6] I. Schur, Uber die Darstellung der endlichen Gruppen durch gebrochene lineare Substitutionen, J. Reine Angew. Math, 127 (1904), 20-50.

[7] I. Schur, Untersuchungen uber die Darstellung der endlichen Gruppen durch gebrochene lineare Substitutionen, J. Reine Angew. Math, 132 (1907), 85-137.

[8] K. I. Tahara, On the second cohomology groups of semidirect products, Math. Z. 129 (1972), 365-379.

[9] L. R. Vermani, An exact sequence, Bull. London Math Soc., 6 (1974), 349-353.

[10] J. Wiegold, Some groups with non-trivial multiplicators, Math. Z. 120 (1971), 307-308.

School of Mathematics, Harish-Chandra Research Institute, Chhatnag Road, Jhunsi, Allahabad 211019, India \& Homi Bhabha National Institute, Training School Complex, Anushakti Nagar, Mumbai 400085, India

E-mail address: sumanahatui@hri.res.in

961, Sector 7, Urban Estate, Kuruskhetra, India

E-mail address: lrver@yahoo.com

School of Mathematics, Harish-Chandra Research Institute, Chhatnag Road, Jhunsi, Allahabad 211019, InDia \& Homi Bhabha National Institute, Training School Complex, Anushakti NAGAR, Mumbai 400085, India

E-mail address: myadav@hri.res.in 